\section{(2) OPEN ACCESS}

\title{
Ethnically diverse urban transmission networks of Neisseria gonorrhoeae without evidence of HIV serosorting
}

\author{
Jayshree Dave (D) ${ }^{1}$ John Paul, ${ }^{2,3}$ Thomas Joshua Pasvol, ${ }^{4}$ Andy Williams, ${ }^{4}$ \\ Fiona Warburton, ${ }^{5}$ Kevin Cole, ${ }^{6}$ Victoria Fotini Miari (1) , ${ }^{7}$ Richard Stabler, ${ }^{7}$ \\ David W Eyre (1) ${ }^{8,9}$
}

${ }^{1}$ National Infection Service, Public Health Laboratory London, Public Health England, London, UK

${ }^{2}$ Department of Global Health and Infection, Brighton and Sussex Medical School, Brighton, UK

${ }^{3}$ Department of Microbiology, Royal Sussex County Hospital, Brighton, UK

${ }^{4}$ Ambrose King Centre, Royal London Hospital, London, UK ${ }^{5}$ Statistics, Modelling and Economics Department, Public Health England, London, UK ${ }^{6}$ Department of Microbiology, Public Health England Collaborative Centre, Royal Sussex County Hospital, Brighton, UK

${ }^{7}$ Department of Infectious and Tropical Diseases, London School of Hygiene \& Tropical

Medicine, London, UK

${ }^{8}$ Nuffield Department of Medicine, University of Oxford, Oxford, UK

${ }^{9}$ Big Data Institute, University of Oxford, Oxford, UK

\section{Correspondence to} Dr David W Eyre, Nuffield Department of Medicine, University of Oxford, Oxford OX3 9DU, UK; david.eyre@ndm. ox.ac.uk

Received 5 March 2019 Revised 24 September 2019

Accepted 6 October 2019 Published Online First 29 October 2019

\section{Check for updates}

(C) Author(s) (or their employer(s)) 2020. Re-use permitted under CC BY-NC. No commercial re-use. See rights and permissions. Published by BMJ.

To cite: Dave J,

Paul J, Pasvol TJ, et al.

Sex Transm Infect

2020:96:106-109.

\begin{abstract}
Objective We aimed to characterise gonorrhoea transmission patterns in a diverse urban population by linking genomic, epidemiological and antimicrobial susceptibility data.

Methods Neisseria gonorrhoeae isolates from patients attending sexual health clinics at Barts Health NHS Trust, London, UK, during an 11-month period underwent whole-genome sequencing and antimicrobial susceptibility testing. We combined laboratory and patient data to investigate the transmission network structure.
\end{abstract}

Results One hundred and fifty-eight isolates from 158 patients were available with associated descriptive data. One hundred and twenty-nine (82\%) patients identified as male and $25(16 \%)$ as female; four (3\%) records lacked gender information. Self-described ethnicities were: 51 (32\%) English/Welsh/Scottish; 33 (21\%) white, other; 23 (15\%) black British/black African/black, other; 12 (8\%) Caribbean; 9 (6\%) South Asian; 6 (4\%) mixed ethnicity; and $10(6 \%)$ other; data were missing for 14 (9\%). Self-reported sexual orientations were 82 (52\%) men who have sex with men (MSM); 49 (31\%) heterosexual; 2 (1\%) bisexual; data were missing for 25 individuals. Twenty-two (14\%) patients were HIV positive. Whole-genome sequence data were generated for 151 isolates, which linked 75 (50\%) patients to at least one other case. Using sequencing data, we found no evidence of transmission networks related to specific ethnic groups ( $p=0.64)$ or of HIV serosorting $(p=0.35)$. Of $82 \mathrm{MSM} /$ bisexual patients with sequencing data, 45 (55\%) belonged to clusters of $\geq 2$ cases, compared with 16/44 (36\%) heterosexuals with sequencing data $(p=0.06)$.

Conclusion We demonstrate links between $50 \%$ of patients in transmission networks using a relatively small sample in a large cosmopolitan city. We found no evidence of HIV serosorting. Our results do not support assortative selectivity as an explanation for differences in gonorrhoea incidence between ethnic groups.

\section{INTRODUCTION}

International travel complicates gonorrhoea transmission and exacerbates the spread of antibiotic resistance. ${ }^{1}$ Technologies such as wholegenome sequencing (WGS) have the potential to revolutionise gonorrhoea diagnosis and treatment in sexual health clinics where repeat attendance is not guaranteed. Previous WGS studies in Brighton, UK, identified transmission networks in a predominantly white British population of men who have sex with men (MSM), revealing clusters with mixed HIV serostatus and links between Brighton, London and the USA. ${ }^{2}$ Among diverse urban populations, STI incidence differences have been reported between ethnic groups. ${ }^{4}$ Such differences have been explained by assortative sexual mixing patterns. ${ }^{5}$ The targeting of public health efforts requires understanding of transmission networks.

Our objective was to combine WGS and epidemiological data to improve our understanding of gonorrhoea transmission networks and antimicrobial resistance in the cosmopolitan population of London.

\section{METHODS}

\section{Setting and participants}

We undertook a retrospective, observational study of unselected patients attending sexual health clinics during two sampling periods (from 20 May 2013 to 16 August 2013 and from 3 December 2013 to 21 March 2014) at Barts Health NHS Trust, London, UK, serving a diverse population with $>100000$ attendances per year of which around $10 \%$ are MSM. Genital and extragenital isolates were collected, according to sexual history, from symptomatic and asymptomatic patients as part of routine care. Information was retrieved regarding: symptoms, age, sex, ethnicity, sexual orientation, HIV and hepatitis B serostatus, recreational/intravenous drug use, number of sexual partners in the last 3 months, sex abroad and nucleic acid amplification test (NAAT) results.

\section{Antimicrobial susceptibility}

Susceptibilities were determined at either of two laboratories, the Royal Sussex County Hospital, Brighton and the London School of Hygiene and Tropical Medicine, using European Committee on Antimicrobial Susceptibility Testing and British Society for Antimicrobial Chemotherapy methods, respectively. Breakpoints used to categorise samples as susceptible and resistant were: azithromycin: 
$\leq 0.25$ and $>0.5$; cefixime: $\leq 0.125$ and $>0.125$; ciprofloxacin: $\leq 0.03$ and $>0.06$; penicillin: $\leq 0.06$ and $>1$; and tetracycline: $\leq 0.5$ and $>1 \mathrm{mg} / \mathrm{L} .{ }^{6}$ Descriptive analyses and Fisher's exact test were undertaken to examine relationships between antimicrobial susceptibilities and risk factors.

\section{Whole-genome sequencing}

One isolate per patient was sequenced. Isolates labelled 'BR-' underwent WGS on the Illumina HiSeq platform as described previously. ${ }^{2}$ Isolates labelled 'LN-' underwent Illumina MiSeq $2 \times 251$ bp paired-end sequencing as per manufacturer's protocol. Sequence data were processed using a bioinformatic pipeline, described previously. ${ }^{2}$ Briefly, sequence reads were mapped to the Neisseria gonorrhoeae NCCP11945 reference genome, and high-quality variants were identified. Samples with $>70 \%$ of the reference genome identified were included in the study. Samples were compared using maximum likelihood phylogenies, ${ }^{7}$ after correction for recombination using ClonalFrameML. ${ }^{8}$ Sequences are available on the NCBI SRA under BioProject PRJNA522696.

\section{RESULTS}

\section{Demographic data}

Data were available for 158 patients. One hundred and twentynine $(82 \%)$ identified as male and $25(16 \%)$ as female; data were unavailable for four patients. One hundred and twenty-three (78\%) individuals were born in Europe (96 (61\%) in the UK), 6 (4\%) Africa, 6 (4\%) South America, 5 (3\%) South Asia, 5 (3\%) South-East Asia/Australia and $4(3 \%)$ in the Caribbean (data unavailable for nine patients). Self-reported recorded ethnicities were: 51 (32\%) English/Welsh/Scottish; 33 (21\%) white, other; 23 (15\%) black British/black African/black, other; 12 (8\%) Caribbean; 9 (6\%) South Asian; 6 (4\%) mixed; and 10 (6\%) other groups (14 data unavailable).

Patients reported their sexual orientation as: 82 (52\%) MSM, $49(31 \%)$ heterosexual and $2(1 \%)$ bisexual (data unavailable for 25 individuals). Regarding HIV status, 22 (14\%) were HIV positive (16 on treatment) and 109 (69\%) were HIV negative; (data unavailable for 27). One hundred and twenty-two (77\%) were hepatitis B negative and $2(1 \%)$ had hepatitis B coinfection (unavailable for 34 patients). Data on recreational drug use were available for 24 patients: 11 (46\%) reported use and 13 (54\%) denied use. No intravenous drug use was recorded.

The number of sexual partners within the last 3 months varied from 1 to 60: 27 patients (17\%) reported one partner, 31 (20\%) two partners, $16(10 \%) 3-4$ partners, 9 (6\%) 5-9 partners and $15(9 \%) \geq 10$ partners (data unavailable for $60(38 \%)$ ). Partner notification occurred for at least 94 (59\%) individuals: information was not available for $42(27 \%)$ and notification did not occur for 22 (14\%) patients. Twelve (8\%) reported sex abroad. One isolate per patient was analysed from the following anatomical sites: 76 (48\%) urethral, 29 (18\%) rectal, 11 (7\%) cervical, $9(6 \%)$ pharyngeal and $33(21 \%)$ not specified. NAAT testing results were available for 109 culture-positive patients; three were NAAT negative.

\section{Antimicrobial susceptibility data}

Antimicrobial susceptibility results were as follows: azithromycin: susceptible 96 (61\%), intermediate $7(4 \%)$, resistant 1 $(1 \%)$ and not available $54(34 \%)$; ciprofloxacin: susceptible 104 $(66 \%)$, intermediate $1(1 \%)$, resistant $37(23 \%)$ and not available $16(10 \%)$; and tetracycline: susceptible $36(23 \%)$, intermediate $19(12 \%)$, resistant $35(22 \%)$ and not available 68 (43\%). All 156 tested isolates were ceftriaxone susceptible (data not available for two). There was no demonstrable relationship between antimicrobial susceptibility for any antibiotic and sexual orientation (Fisher's exact test: $p \geq 0.49$ across all antibiotics) or number of partners $(\mathrm{p} \geq 0.43)$.

\section{Whole-genome sequencing}

WGS data were successfully generated for 151/158 (96\%) isolates. After mapping a median (IQR) (range) 89.1\% (87.7\%89.6\%) (77.8-90.6\%) of the NCCP11945 reference genome was identified. We applied our previously described transmission nomogram ${ }^{2}$ to link cases related by possible direct personto-person transmission or indirect transmission via one or more intermediate cases. Cases were considered genetically linked if the number of single nucleotide polymorphisms (SNPs) between them fell within the $99 \%$ prediction interval for the number expected between transmitted cases based on the time between sampling. Of 151 cases, 76 (50\%) were not linked to another case, $30(20 \%)$ belonged to potential transmission pairs, $39(26 \%)$ triplets and 6 cases (4\%) formed the largest cluster (figure 1). Overall, 105 distinct genetic subtypes of $N$. gonorrhoeae were observed. The largest cluster spanned June 2013 to March 2014 and consisted of four MSM, one heterosexual male and a male with undisclosed sexual orientation. All six men were born in Europe (four in the UK) and from varying ethnic groups: white, South Asian and mixed ethnicity. One was HIV positive (treated); another had chronic hepatitis $\mathrm{B}$ infection.

Figure 1 shows all sequenced isolates with patient information. We investigated for evidence of genetic clustering of cases by reported sexual orientation (as a positive control), HIV serostatus and ethnicity. We considered the number of pairs of cases within genetic clusters who shared the same characteristic and compared this with the distribution when we randomly permuted the characteristic labels 1000 times. Forty-one within-cluster pairs had the same reported sexual orientation and seven had different orientations; 21 pairs had missing data. Under the permutated model, representing the distribution expected by chance, many more within-cluster pairs had different sexual orientations, median 23 pairs $(95 \%$ CI 14 to 30); that is, there is strong evidence for clustering by sexual orientation as the number of observed pairs with different sexual orientations was much lower than the lower bound of the permutation CI.

However, there was no evidence of HIV serosorting beyond that expected by chance: 34 potential transmission pairs had the same HIV serostatus and 12 pairs differed; data were missing for 23 pairs. This compares with 17 discordant pairs $(95 \% \mathrm{CI}$ 10 to 23 , ie, containing 12) under the random model. All HIVpositive patients with available data identified as MSM $(n=21)$ or bisexual $(n=1)$. Similarly, within the limits of the power of the study, there was no evidence for clustering by ethnic group, 15 pairs shared the same ethnic group, 45 a different ethnic group, and data were missing for nine pairs. Forty-five $(95 \%$ CI 36 to 52) discordant pairs were expected under the random permutation model.

Overall proportions of cases involved in any cluster were similar by ethnic group (Fisher's exact test: $p=0.64$ ) and HIV serostatus $(p=0.35)$. Forty-five out of $82(55 \%)$ MSM or bisexual clients with sequencing data were in genetic clusters of $\geq 2$ cases, compared with 16/44 (36\%) of clients identifying as heterosexual $(\mathrm{p}=0.06)$. Fifty-six per cent $(14 / 25)$ patients not recording their sexuality were part of potential transmission clusters. 

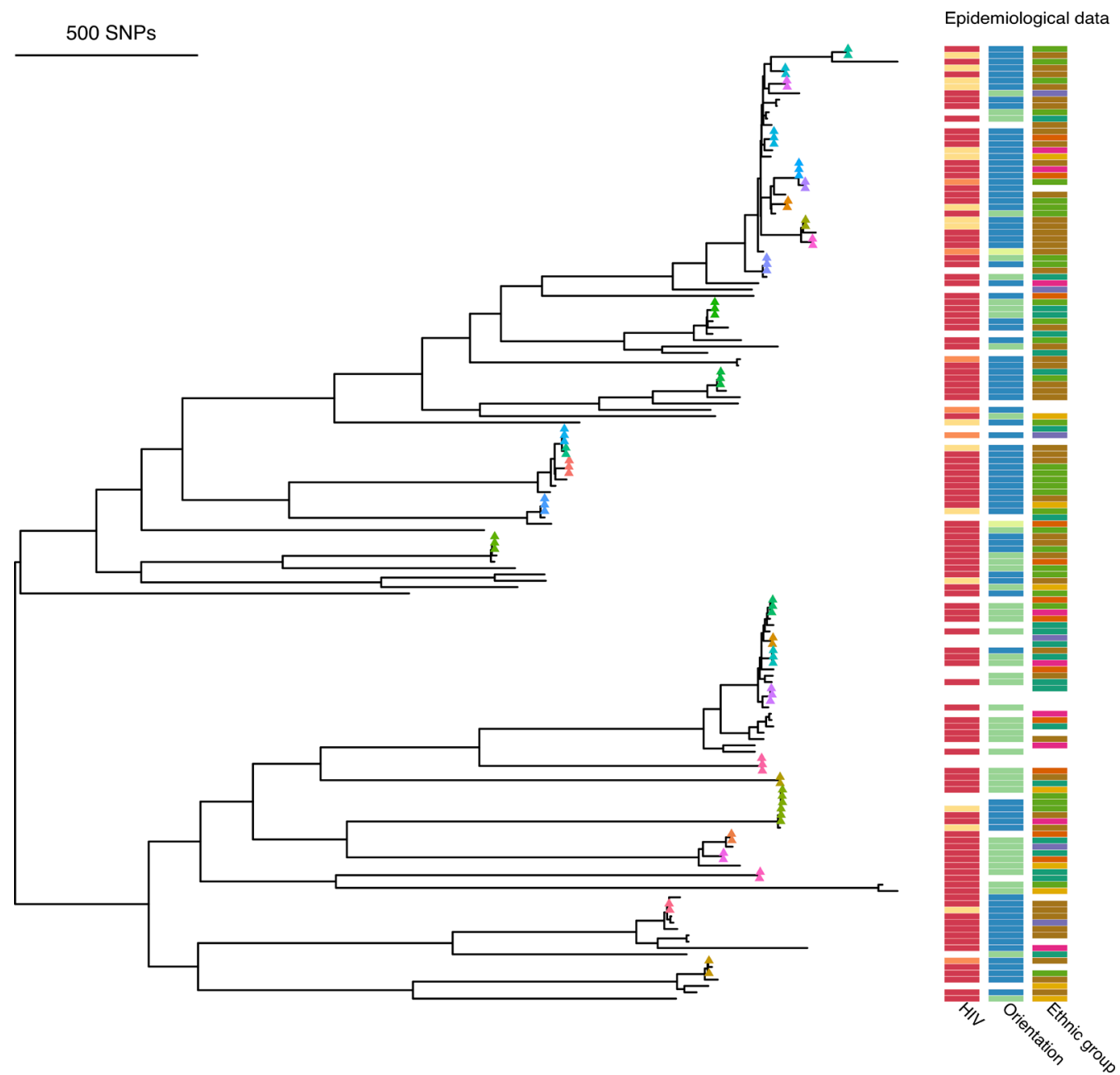

Epidemiological data legend

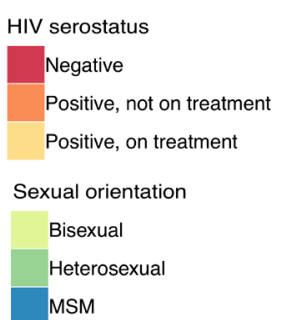

Ethnic Group

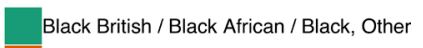
Caribbean Mixed Other White, Other South Asian English / Scottish / Welsh

Figure 1 Phylogeny of 151 Neisseria gonorrhoeae isolates from London, May 2013-March 2014. Tips with coloured triangles represent potential transmission clusters, based on the genetic distance and time between isolates. ${ }^{2}$ The colouring of each transmission cluster is arbitrary. The right-hand panels show associated epidemiological data regarding HIV serostatus, sexual orientation and self-reported ethnic group. MSM, men who have sex with men.

\section{DISCUSSION}

Using combined WGS and epidemiological data, we investigated gonorrhoea in a diverse urban population. Previous studies ${ }^{4}$ demonstrated variation in gonorrhoea incidence within different ethnic groups. We detected no evidence of clustering by ethnicity. Therefore, our findings do not support the hypothesis that infection rate differences between ethnic groups can be explained by assortative sexual mixing. However, our study was limited by the relatively small sample size, with only 151 isolates sequenced.

We demonstrated increased linkage of cases among MSM, and we detected clusters involving MSM of mixed HIV serostatus, as reported previously ${ }^{3}$ and contrary to the findings of a study using samples from 2004. ${ }^{9}$ We demonstrate bridging events between different ethnicities and sexual orientation. We found less case clustering than in Brighton. ${ }^{3}$ This may reflect less complete sampling, as London is served by multiple clinics and less clustering of cases in the non-MSM population.

Our results show evidence of transmission in heterosexuals and in MSMs of different ethnicity. As in Brighton, we found evidence of gonorrhoea transmission involving patients of mixed HIV serostatus. Decreased concern regarding HIV risks may be contributing to increased condomless sex and gonorrhoea transmission. The impact on transmission networks of the wider availability of HIV pre-exposure prophylaxis since this study could be investigated using WGS in future.

Handling editor Henry John Christiaan de Vries

Twitter Victoria Fotini Miari @FusilliTeeth

Acknowledgements We thank Glenn Phiri for administrative support.

Contributors Designed the study: JD, JP, DWE, RS and AW. Collected patient data and isolates: AW and TJP. Processed the isolates: VFM and KC. Performed bioinformatic analysis: RS and DWE. Analysed the data: JD, JP, RS, FW and DWE. Prepared the figure: DWE. Wrote the manuscript: JD, JP, DWE, FW and RS. Revision of manuscript: all authors.

Funding The research was funded by the National Institute for Health Research Health Protection Research Unit (NIHR HPRU) in healthcare-associated infections and antimicrobial resistance at the University of Oxford in partnership with Public Health England (PHE) (HPRU-2012-10041) and the NIHR HPRU in Modelling Methodology at Imperial College London (HPRU-2012-10080) in partnership with PHE. DWE is a Big Data Institute Robertson Fellow.

Competing interests None declared.

Patient consent for publication Not required.

Provenance and peer review Not commissioned; externally peer reviewed.

Open access This is an open access article distributed in accordance with the Creative Commons Attribution Non Commercial (CC BY-NC 4.0) license, which permits others to distribute, remix, adapt, build upon this work non-commercially, and license their derivative works on different terms, provided the original work is 
properly cited, appropriate credit is given, any changes made indicated, and the use is non-commercial. See: http://creativecommons.org/licenses/by-nc/4.0/.

\section{ORCID iDs}

Jayshree Dave http://orcid.org/0000-0002-5689-7975

Victoria Fotini Miari http://orcid.org/0000-0002-5995-9422

David W Eyre http://orcid.org/0000-0001-5095-6367

\section{REFERENCES}

1. Wi T, Lahra MM, Ndowa F, et al. Antimicrobial resistance in Neisseria gonorrhoeae: Global surveillance and a call for international collaborative action. PLoS Med 2017; 14:e1002344.

2. De Silva D, Peters J, Cole K, et al. Whole-genome sequencing to determine transmission of Neisseria gonorrhoeae : an observational study. Lancet Infect Dis 2016;16:1295-303.

3. Peters J, Cresswell F, Amor L, et al. Whole genome sequencing of Neisseria gonorrhoeae reveals transmission clusters involving patients of mixed HIV serostatus. Sex Transm Infect 2018:94:138-43.
4. Hulstein SH, Matser A, Alberts CJ, et al. Differences in Chlamydia trachomatis seroprevalence between ethnic groups cannot be fully explained by socioeconomic status, sexual healthcare seeking behavior or sexual risk behavior: a cross-sectional analysis in the Healthy Life in an Urban Setting (HELIUS) study. BMC Infect Dis 2018; $18: 612$.

5. Low N, Sterne J, Barlow D. Inequalities in rates of gonorrhoea and chlamydia between black ethnic groups in South East London: cross sectional study. Sex Transm Infect 2001;77:15-20.

6. EUCAST. Clinical Breakpoints-Bacteria (v6.0). 2016. The European Committee on antimicrobial susceptibility testing. breakpoint tables for interpretation of MICs and zone diameters. version 6.0, 2016. Available: http://www.eucast.org/fileadmin/src/ media/PDFs/EUCAST files/ Break point tables/v 6.0. Breakpoint table.pdf

7. Guindon $S$, Lethiec F, Duroux $P$, et al. PHYML Online--a web server for fast maximum likelihood-based phylogenetic inference. Nucleic Acids Res 2005;33:W557-9 https:// doi.org/

8. Didelot $X$, Wilson DJ. ClonalFrameML: efficient inference of recombination in whole bacterial genomes. PLoS Comput Biol 2015;11:e1004041.

9. Didelot X, Dordel J, Whittles LK, et al. Genomic analysis and comparison of two gonorrhea outbreaks. MBio 2016;7:e00525-16. 